\title{
How Does the Number of Arms Affect the Properties of Mikto-Arm Stars in a Selective Oligomeric Matrix? Insights from Atomistic Simulations
}

Petra Bačová,* Emmanouil Glynos, Spiros H. Anastasiadis, and Vagelis Harmandaris*

Cite This: ACS Omega 2021, 6, 1138-1148

Read Online

S1 Supporting Information

ABSTRACT: We present a simulation study of amphiphilic mikto-arm star copolymers in a selective polymer host. By means of atomistic molecular dynamics simulations, we examine the structural and dynamical properties of mikto-arm stars with varying number, $n$, of poly(ethylene oxide) (PEO) and polystyrene (PS) arms, (PEO) $)_{n}(\mathrm{PS})_{n}$ in a $33 \%$ wt blend with an oligomeric PEO host (o-PEO). As the number of arms increases, the stars resemble more spherical particles with less separated PEO and PS intramolecular domains. As a result of their internal morphology and associated geometrical constraints, the mikto-arm stars self-assemble either into cylindrical-like objects or a percolated network with increasing $n$, within the o-PEO matrix. The segmental dynamics is mostly governed by the star architecture and the

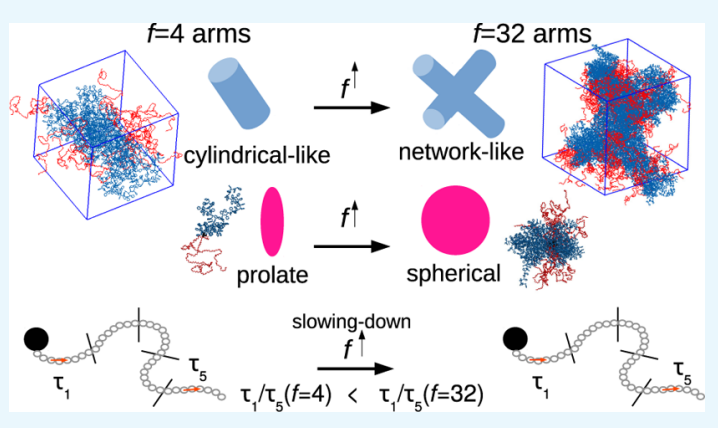
heterogeneous local environment, formed by the intra- and intermolecular nanosegregation. We discuss the role of each factor and compare the results with previously published studies on mikto-arm stars.

\section{INTRODUCTION}

The high demand on versatile products pushes the material scientist to come up with materials which combine various desired properties in one entity. ${ }^{1,2}$ In this effort, the bottom-up approach integrates multiphase building blocks, such as Janus or patchy particles, into superstructures with nanosegregated domains. ${ }^{3-8}$ As an example, a variety of nanostructured particles have been formed using triblock copolymers and tuning the mutual interactions in the sample in a sequel of steps. ${ }^{9-11}$ These all-polymer, building blocks prepared by a directed selfassembly, in contrast to their analogues consisting of surfacemodified inorganic nanoparticles, such as functionalized hairy silica nanoparticles, ${ }^{12-15}$ exhibit not only contrasting surface properties but also an intrinsic anisotropy. ${ }^{16,17}$

Among the various nanosegregated nano-objects, star-shaped copolymers have a clear advantage of being multicomponent molecules with a well-defined architecture. By selecting the polymer components, as well as adjusting the star composition under given conditions, one is able to obtain a huge family of intramolecularly nanostructured objects and, consequently, a huge variety of self-assembled structures. ${ }^{7,18-26}$

Concerning copolymer stars composed of heteroarms (the socalled mikto-arm stars), studies on how a specific polymer host guides their nanosegregation processes are very scarce. Some experimental studies have explored the regime of stars with a low number of arms (i.e., with low functionality) and/or dilute conditions. ${ }^{27-33}$ Similarly, simulation studies focus almost exclusively on the dilute regime, investigating properties such as the aggregation number, while varying the length of the star arm and/or solvent quality. ${ }^{34-38}$ Simulation techniques, using generic bead-spring models, proved to be useful when investigating conformational properties of single-molecule mikto-arm stars. More specifically, Monte Carlo ${ }^{18,39-41}$ and dissipative particle dynamics ${ }^{42}$ were used to examine factors such as the solvent quality or the mutual interaction between the arms, by changing the parameters of the coarse-grained model. Concerning the intramolecular segregation, Chang et al. ${ }^{18}$ studied mikto-arm stars with $f \leq 16$ under various solvent conditions. They quantified the degree of segregation for Januslike arrangement and reported its nonmonotonic dependence on the number of arms in the case of a very strong repulsion between the two star components. By evaluating the potential of mean force, they also concluded that in the case of a selective solvent, one component segregates internally into "globule" and the final interaction between two mikto-arm stars is determined by the competition between the attraction of these "globule" regions and the excluded-volume repulsions.

When dealing with heterogeneous materials, chemistryspecific characteristics, such as stiffness or local packing, may be crucial when determining the nanosegregation processes. To address this challenge, we have recently employed an atomistic

Received: August 28, 2020

Accepted: November 17, 2020

Published: January 4, 2021 

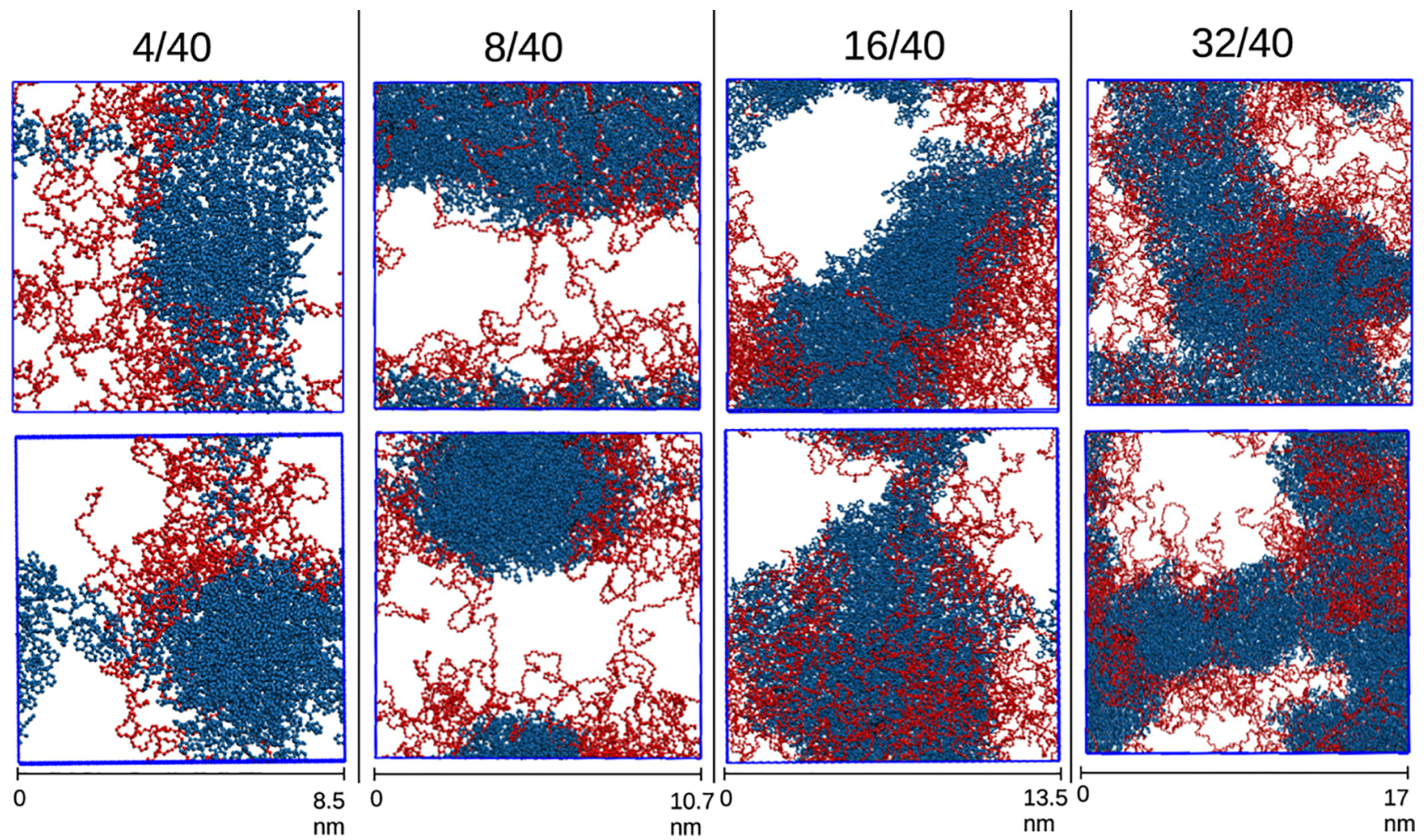

Figure 1. Representative snapshots of assembled stars. Top row: side view; bottom row: front view. The atoms belonging to the PEO arms are painted in red and to the PS arms are painted in blue. The o-PEO matrix is omitted for clarity. The numbers at the bottom indicate the average cubic box size for each system.

simulation method to develop a bottom-up modeling approach to design nanostructured materials, which integrates the features of mikto-arm stars consisting of structurally and dynamically contrasting polymers. More specifically, we turned our attention on mikto-arm stars with an equal number of immiscible polystyrene (PS) and poly(ethylene oxide) (PEO) arms. Considering the single-star properties, we showed that by attaching immiscible PS and PEO arms into a star-like architecture, the mikto-arm stars nanosegregate intramolecularly into particles with Janus-like, patchy-like, or octopus-like patterns. ${ }^{25}$ The rich internal morphology, imposed by the number of star arms (i.e., functionality $f$ ) and/or the given environment, earns them a special place in the family of nanosegregated particles, with a potential to be used as versatile soft building blocks. ${ }^{25}$ Because of the large difference in the glass transition temperatures of the PEO and PS arms (more than 150 $\mathrm{K}^{43}$ ), nanosegregated domains rich in one of these two components exhibit different dynamical behaviors. ${ }^{44}$ As the arrangement of the domains is constrained by a star-like architecture, the segmental dynamics of the different components, in such a heterogeneous environment, is strongly correlated. ${ }^{44}$ Highlighting the role of star-like architecture in self-assembly processes, we compared $\left(\mathrm{PEO}_{80}\right)_{8}\left(\mathrm{PS}_{80}\right)_{8}$ stars and their linear $\left(\mathrm{PEO}_{80}\right)\left(\mathrm{PS}_{80}\right)$ block copolymer analogues, both blended at the weight fraction $33 \%$ with an oligomeric PEO matrix. ${ }^{24}\left(\mathrm{PEO}_{80}\right)_{8}\left(\mathrm{PS}_{80}\right)_{8}$ stars with 16 arms and 80 mers per arm formed percolated networks, in a qualitative agreement with an experimental study of similar systems of mikto-arm stars. ${ }^{26}$

Following and building up on the abovementioned observations, we focus, in this work, on one complex missing piece of the puzzle: how the functionality (i.e., the number of arms), in combination with chemistry-specific interactions, which are driving the self-assembly process, affects the properties of the final nanostructured material. Keeping in mind that the number of arms (functionality $f=2 n$ ) and the local environment govern the internal nanosegregation, ${ }^{25}$ functionality is the only tuning parameter in the current work. The length of the arms $m$, the star weight fraction in the oligomeric PEO matrix, and the temperature are kept constant. In order to partially rule out the effect of the functionality and to consider the self-assembly behavior of these systems closely, we also use data from singlestar simulations of analogous stars, introduced in ref 25 , as reference systems. In addition, when the piece of information contributes to a better understanding of the problem and helps to create a compact picture, we confront the results with our former study on star systems with longer arms in the same selective oligomeric matrix. ${ }^{24}$ We discuss the self-assembly of the stars in a selective medium, their individual conformations in the self-assembled object, and the dynamical heterogeneities stemming from the star-like architecture and the local environment.

\section{RESULTS AND DISCUSSION}

2.1. Static Properties. 2.1.1. Self-Assembly Behavior. We simulated four blends of mikto-arm stars with different functionalities, $f$, within a selective homopolymer matrix. More specifically, the blends consisted of $10\left(\mathrm{PS}_{m}\right)_{n}\left(\mathrm{PEO}_{m}\right)_{n}$ stars, each having $n$ arms of PS and $n$ arms of PEO, where $f=2 n$. The arms are attached alternately to a dendritic core (see the scheme in Figure 9 in Section 4). The length of each arm is $m=40$ monomeric units, and we modeled stars with $f=4,8,16$, and 32 . We use a notation $\mathrm{f} / \mathrm{m}$ to distinguish the systems, that is, the notation 32/40 means a blend of $\left(\mathrm{PS}_{40}\right)_{16}\left(\mathrm{PEO}_{40}\right)_{16}$ stars in an oligomeric PEO (o-PEO) host. The oligomeric PEO chains 

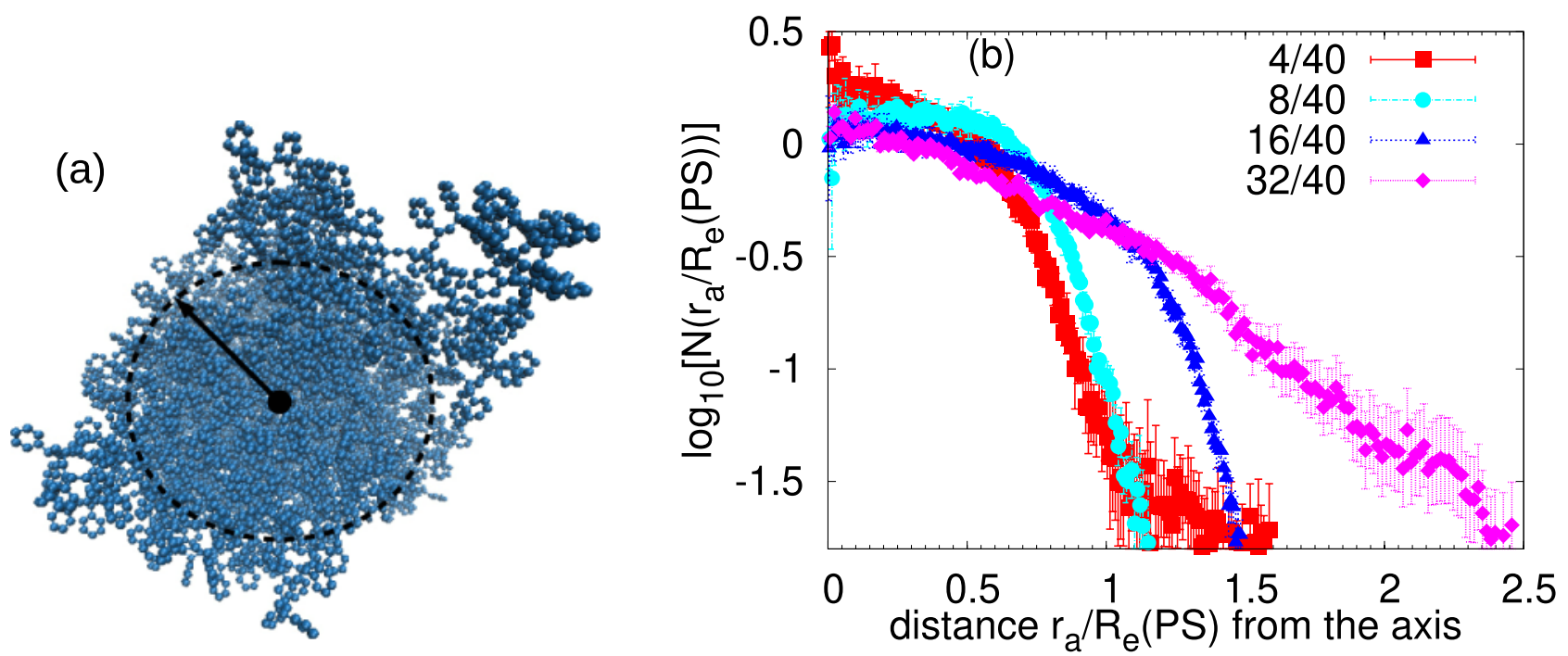

Figure 2. (a) Schematic illustration of the projection of positions of the atoms on PS arms (blue) in the assembly (front view) in the $8 / 40$ system into the radial profile. The black dot represents the main axis and the arrow one of the selected distances $r_{\mathrm{a}}$ of an atom from the axis. (b) Probability $N\left(r_{\mathrm{a}} /\right.$ $R_{\mathrm{e}}(\mathrm{PS})$ ) of finding an atom in the distance $r_{\mathrm{a}} / R_{\mathrm{e}}$ (PS) from the center of assembly, defined by the main axis of the radius of gyration tensor. $R_{\mathrm{e}}$ (PS) corresponds to the root mean square value of the center-to-end vector of PS arms. The error bars were estimated from the standard block averaging method analysis.

Table 1. Radii of Gyration of the Stars $\left(R_{\mathrm{g}}\right)$, the Root Mean-Squared Values of the Center-to-End Vectors $\left(R_{\mathrm{e}}\right)$ of the Star Arms, and Values of the Shape Parameters, Asphericity $a$ and Prolateness $p$

$\begin{array}{lcc}\text { system } & R_{\mathrm{g}}(\mathrm{nm}) & R_{\mathrm{e}}(\mathrm{PEO})(\mathrm{nm}) \\ 4 / 40 & 2.51 \pm 0.07 & 4.2 \pm 0.2 \\ 8 / 40 & 2.71 \pm 0.01 & 4.3 \pm 0.1 \\ 16 / 40 & 2.85 \pm 0.02 & 4.38 \pm 0.08 \\ 32 / 40 & 3.29 \pm 0.01 & 4.91 \pm 0.05\end{array}$

consist of 10 monomeric units, and the number of chains in the homopolymer matrix was adjusted in order to have $33 \%$ weight fraction of the mikto-arm stars in the blend, for all systems.

In pursuit of a bottom-up computational design, we use systems consisting of a single star with the same characteristics (i.e., the same $f$ and $m$ ) in a box with the PEO matrix as reference systems. The details about these single-star simulations can be found elsewhere. ${ }^{25}$ To avoid confusion, we refer to these systems as "single-star" simulations and to those with 10 stars in a box as "multiple-star" simulations. In order to get a hint on a possible effect of the arm length, we refer, in some occasions, to our former study on $\left(\mathrm{PEO}_{80}\right)_{8}\left(\mathrm{PS}_{80}\right)_{8}$ stars in the o-PEO host. ${ }^{24}$ In such a case, we use the same notation $(f / m)$ with the corresponding reference, that is, $16 / 80,{ }^{24}$ in order to refer the reader for further information about properties not presented here. The comparison is always made at the temperature $400 \mathrm{~K}$ and the same weight fraction of stars of $33 \%$. Moreover, the preparation and the used force field in ref 24 are identical with the present work, ruling out technical details as a source of discrepancies.

Representative snapshots from the simulations are displayed in Figure 1, where the PS arms are shown in blue and PEO arms are shown in red. For a better visibility, the o-PEO host molecules were removed. The final morphology of the studied polymer material is governed by the mutual interactions among the system components. The PS arms in the amphiphilic stars tend to minimize the number of contacts with the selective (bad "solvent") environment, that is, with the PEO arms and the oligomeric matrix. This tendency leads to the formation of selfassembled objects, with a central part consisting of PS monomer

$\begin{array}{ccc}R_{\mathrm{e}}(\mathrm{PS})(\mathrm{nm}) & a & p \\ 3.60 \pm 0.06 & 0.24 \pm 0.01 & 0.4 \pm 0.1 \\ 3.55 \pm 0.08 & 0.105 \pm 0.005 & 0.23 \pm 0.02 \\ 3.49 \pm 0.02 & 0.068 \pm 0.006 & 0.39 \pm 0.06 \\ 3.68 \pm 0.04 & 0.026 \pm 0.001 & 0.14 \pm 0.05\end{array}$

units. The PEO arms, as expected, are oriented toward the polymer matrix, forming "hairs" around the central part of the assembly. Noticeably, the shape of the assembled object depends on the functionality of mikto-arm stars. Although the stars with low functionality $(f \leq 16)$ form cylindrical objects, the mikto-arm stars with $f=32$ segregate into a percolated network.

In order to characterize the morphology of the assemblies, we generate a radial profile of the atoms of PS arms via the following procedure: we first calculate the radius of gyration tensor of the entire self-assembled object, and then, we define the main axis of the object as the axis corresponding to the vector with the largest eigenvalue of the radius of gyration tensor (see the Supporting Information for details about the radius of gyration tensor). Afterward, we measure all atom distances, $r_{a}$, for the atoms of PS arms from the main axis of the assembly and analyze their distribution in radial shells, as it is illustrated in Figure 2a. This allows us to project the positions of atoms in the assembly into a radial $2 \mathrm{D}$ profile with the main axis being the central point. The probability distributions of the rescaled distances $N\left(r_{\mathrm{a}} / R_{\mathrm{e}}(\mathrm{PS})\right)$, where $R_{\mathrm{e}}$ (PS) denotes the (average) magnitude of the center-toend vector of the PS arm, measured from the arm attachment point to the arm tip (its values are listed in Table 1 ), are shown in Figure $2 \mathrm{~b}$. The shape of the distributions for the $4 / 40$ and $8 / 40$ systems, with a final sudden drop, is characteristic for a cylindrical-like object, where the atoms are mostly located around the main axis and after a certain distance, which would correspond to the radius of the cylinder, the probability of finding an atom drops significantly for larger distances. For both $4 / 40$ and 8/40 systems, the sudden decrease in $N\left(r_{\mathrm{a}} / R_{\mathrm{e}}(\mathrm{PS})\right)$ occurs at around $r_{\mathrm{a}} \approx R_{\mathrm{e}}(\mathrm{PS})$; however, the packing of PS arms 

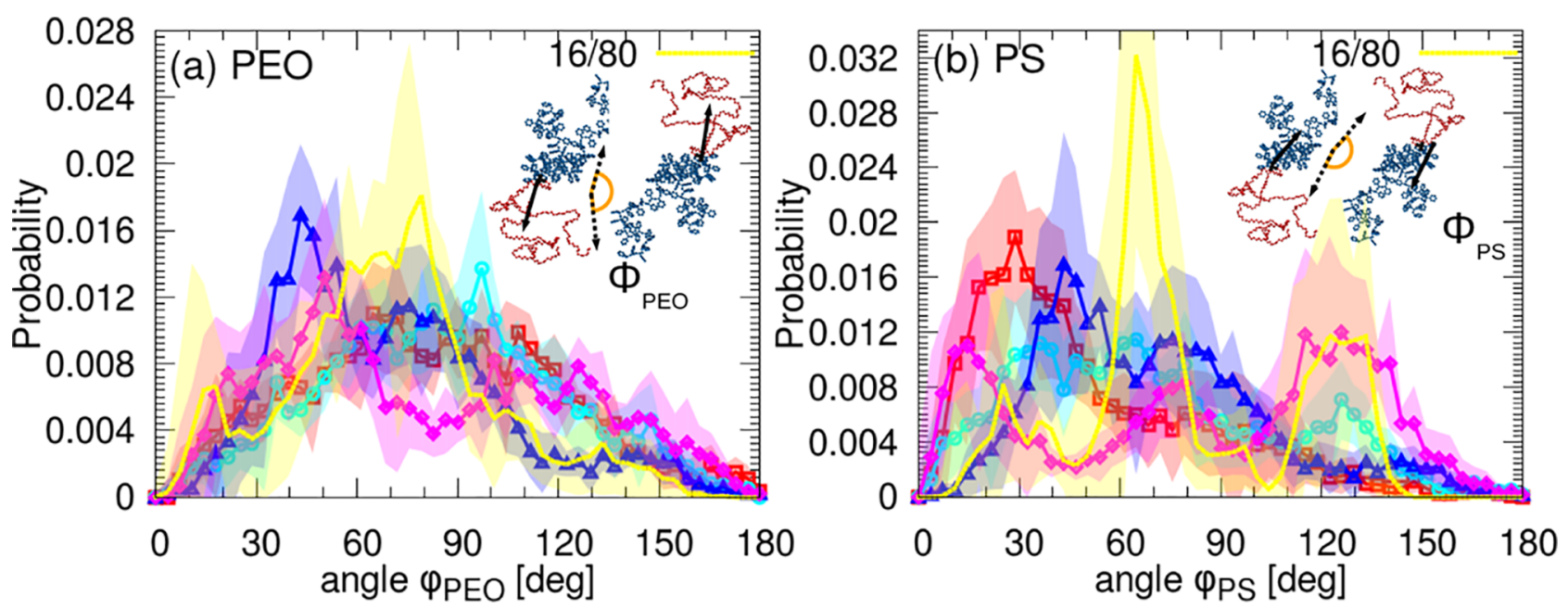

Figure 3. Angle distribution for (a) $\phi_{\mathrm{PEO}}$ and (b) $\phi_{\mathrm{PS}}$ in the simulated systems: $4 / 40$ (red squares), 8/40 (cyan circles), 16/40 (blue triangles), and 32/ 40 (magenta diamonds). $\phi_{\mathrm{PEO}}$ and $\phi_{\mathrm{PS}}$ are angles between the vectors in the nearest neighbor stars; the vectors connect the middle carbon of the star core and the center-of-mass of the PEO and of the PS component, respectively, as illustrated in the inset. The error bars estimated from the standard block averaging method analysis are shown as the shaded area.
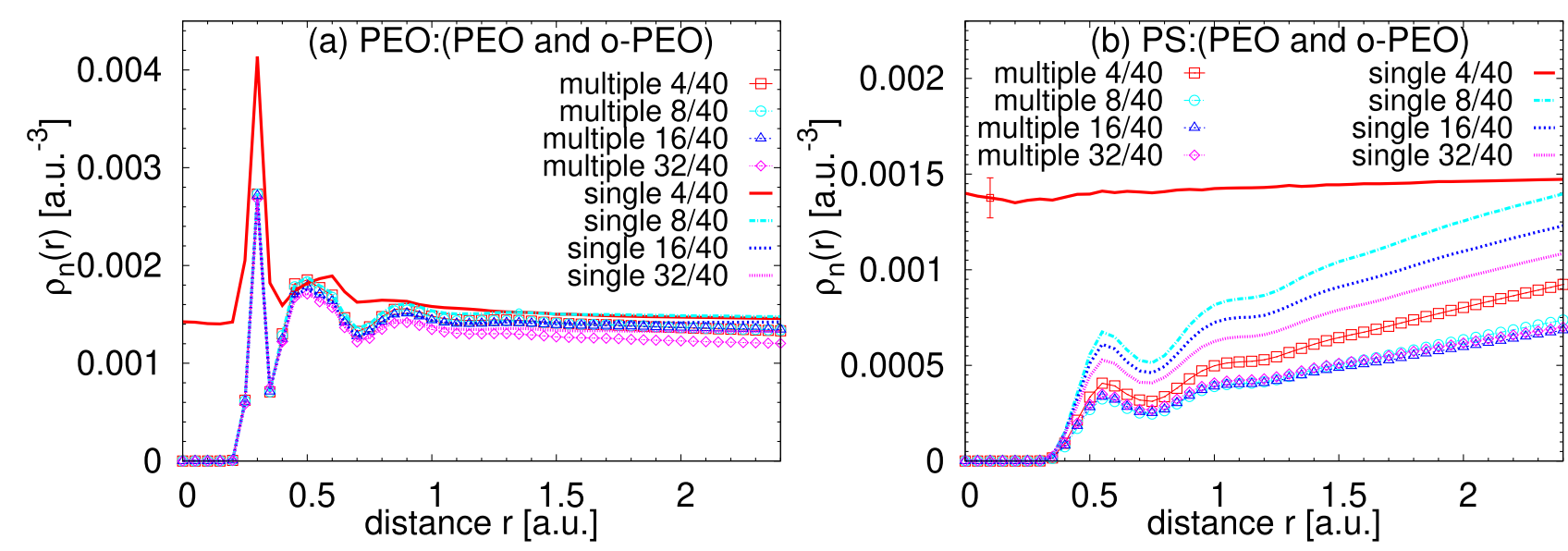

Figure 4. Local number density $\rho_{\mathrm{n}}(r)$ for all PEO monomers in the system (i.e., the PEO monomers of the star arms and o-PEO) as a function of the distance $r$ from (a) the PEO and (b) the PS monomers on the star arms. The symbols correspond to the multiple-star simulations, and the lines correspond to the single-star simulations. The biggest error bar estimated from the standard block averaging method analysis is shown for $4 / 40$ singlestar simulations. The error bars of the remaining systems are of the order of the symbol size or of the line thickness.

in the cylindrical assembly seems to be more efficient in systems with $f=8$, where the constant value of $N\left(r_{\mathrm{a}} / R_{\mathrm{e}}(\mathrm{PS})\right)$ at distances $r_{\mathrm{a}}<0.7 R_{\mathrm{e}}$ (PS) points out to a homogeneous radial profile within the assembly. The tail in the probability distribution function for $4 / 40$ is caused by a small protrusion in the assembled object, which can be spotted by a closer inspection of Figure 1 . The distribution for $16 / 40$ is wider, with a smoother decay, identifying a cylindrical-like object with either fuzzier borders or with a varying radius. The much more isotropic percolated network formed by the PS arms of mikto-arm stars with $f=32$ gives rise to a stretched distribution, with a nonzero probability of finding an atom at distances much larger than the star size (the root mean square values of the star radius of gyration are listed in Table 1). When rescaled with the corresponding $R_{\mathrm{e}}(\mathrm{PS}), N\left(r_{\mathrm{a}} /\right.$ $R_{\mathrm{e}}$ (PS)) reported for 16/80 mikto-arm stars ${ }^{24}$ (not shown here) closely matches the distribution for $32 / 40$ stars studied in this work. Note that both $16 / 80$ and $32 / 40$ systems self-assemble into a network-like structure in the o-PEO matrix at $400 \mathrm{~K}$. Seemingly, both factors, the functionality and the arm length of the segregated stars, determine the final morphology of the selfassembled objects.

To investigate the spatial arrangement of the stars in the assembly, we measure the angle between the vectors connecting the star centers and the center-of-mass of the given polymer star component in the nearest neighbor stars. Namely, for each star, we define a vector $\mathbf{R}_{\mathrm{s}}=\mathbf{r}_{\mathrm{cm}, \mathrm{s}}-\mathbf{r}_{0}$, where $\mathbf{r}_{0}$ is the position of the central carbon atom in the core (black square in Figure 9) and $\mathbf{r}_{\mathrm{cm}, \mathrm{s}}$ is the position of the center-of-mass of the polymer type $\mathrm{s}$ (i.e., either PEO or PS). The center-of-mass for each $s$ was found by including all atoms of the given type in the star, that is, all atoms of the PEO or PS arms. Then, the angle between the vectors corresponding to the same polymer type, but lying on the nearest neighbor stars, was measured, see the inset of Figures $3 \mathrm{a}, \mathrm{b}$ and $\mathrm{S} 1$ in the Supporting Information. The normalized distributions of the intermolecular angles $\phi_{\text {PEO }}$ plotted in Figure 3a for mikto-arm stars with $f=4$ and $f=8$ are broad, with no preferential mutual orientation of the PEO domains. As the functionality $f$ increases, the distributions exhibit clear maxima, indicating that the "hair" coverage (i.e., the way the PEO arms 

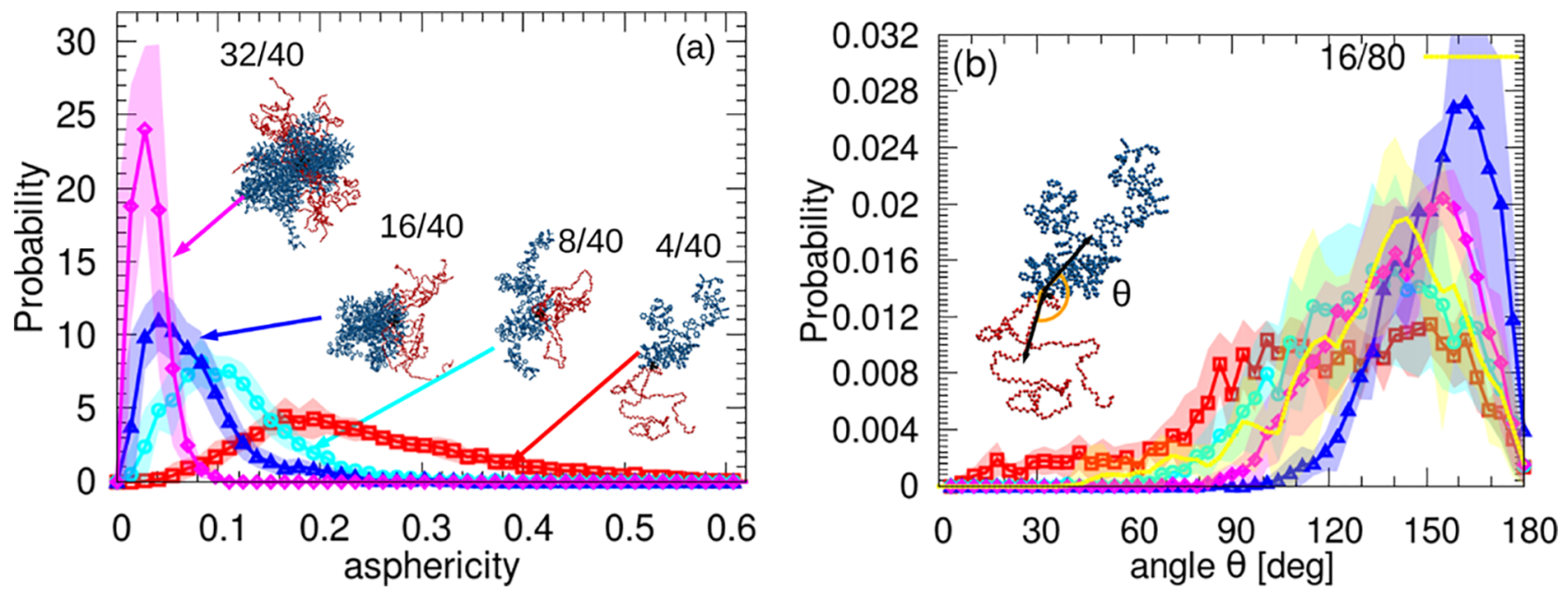

Figure 5. (a) Distributions of the asphericity parameter of individual stars in the assembly. The snapshots represent a characteristic configuration of an individual star of the multiple-star system. (b) Normalized distributions of intramolecular angles $\theta$ (see the inset) for the simulated systems: $4 / 40$ (red squares), 8/40 (cyan circles), 16/40 (blue triangles), and 32/40 (magenta diamonds). The error bars estimated from the standard block averaging method analysis are shown as the shaded area.

arrange around the PS assembly) is not random. The distributions of $\phi_{\mathrm{PS}}$ angles elucidate the packing of the PS domains with respect to their closest neighbors inside of the assembly. In cylindrical-like assemblies, that is, systems $4 / 40$, $8 / 40$, and $16 / 40$, most of the stars are aligned next to each other, with a preferential angle $\phi_{\mathrm{PS}}<90^{\circ}$. The main maximum in these distributions moves to higher values of angles $\phi_{\mathrm{PS}}$ as the functionality increases. The mikto-arm stars $32 / 40$ forming the percolated network exhibit a distribution of $\phi_{\mathrm{PS}}$ with multiple maxima. Interestingly, the $\phi_{\mathrm{PEO}}$ distribution for $16 / 80$ stars $^{24}$ resembles the shape of the distribution corresponding to $16 / 40$ in the current study (compare the yellow dashed line with blue up-pointing triangles in Figure $3 \mathrm{a})$, while the $\phi_{\mathrm{PS}}$ distribution has almost identical positions of the peaks with $32 / 40$ (compare the yellow dashed line with magenta diamonds in Figure $3 b$ ). However, the two network-like assemblies (i.e., the assemblies formed by $16 / 80$ and $32 / 40$ stars) are characterized by distributions of $\phi_{\mathrm{PS}}$ which differ in the amplitudes of the given maxima.

The interplay between intermolecular attraction of PS arms, which promotes aggregation, and the athermal interactions of PEO arms with the o-PEO host in the self-assembled structures can also be examined by the local number density, $\rho_{\mathrm{n}}(r)$, around the specific type of monomers. In Figure 4, the $\rho_{\mathrm{n}}(r)$ centered in the PEO and PS arm monomers is plotted. Figure 4a shows that the local environment around the PEO star monomers is very similar for all studied systems, except for the single-star 4/40 simulations, because of the fully penetrable character of the mikto-arm star with four arms. For the stars with the functionality $f=32$, the probability of finding a PEO monomer fully surrounded by the monomers of the same type is slightly lower because of the associated geometric constraints (notice the systematically lower values of $\rho_{\mathrm{n}}(r)$ for 32/40 in Figure 4a).

The local environment around the PS monomers, shown in Figure $4 \mathrm{~b}$, differs for the single-star and multiple-star simulations, as expected, because the only way to avoid unfavorable PS/PEO interactions in the single-star simulations is to segregate intramolecularly, and thus, the higher the number of arms, the lower the probability of finding a PEO monomer around the PS arms. Again, the fully penetrable character of the $4 / 40$ single-star system is evident. All different $\rho_{\mathrm{n}}(r)$ curves around the PS monomers in the multiple-star simulations are always below the data for the single-star simulations because PS/ $\mathrm{PEO}$ contacts can be further reduced by forming rich PS regimes involving more than one mikto-arm star. This observation suggests a more "homogeneous" environment for the PS arms segregated into an assembly, that is, the PS monomers are mostly surrounded by the monomers of the same type in the given self-assembled object. The data for multiple-star systems with $f>4$ overlap, only the local number density of $4 / 40$ stars deviates, which may be attributed to the higher penetrability of 4/40 stars together with the slightly asymmetrical character of the assembly (see Figure $2 b$ ).

2.1.2. Properties of Individual Stars within the SelfAssembled Structures. We proceed with the analysis of conformational properties of individual stars within the selfassembled structure. We calculated two shape parameters: asphericity $a$ and prolateness $p$, and their definitions can be found in the Supporting Information. Their mean values for each mikto-arm star are listed in Table 1 . The probability distribution functions of $a$ are shown in Figure 5a together with a snapshot of the corresponding star. The higher the star functionality, the more spherical is the shape of the mikto-arm star within the assembly (i.e., the lower the values of the asphericity parameter). Interestingly, the mean value of asphericity for the star with the functionality $8(a=0.105 \pm$ 0.005 ) is in a range of values obtained for a coarse-grained model of an amphiphilic star with eight arms of equal length. ${ }^{42}$ Concerning the shape changes of stars when self-assembling, no differences in shape for mikto-arm stars with $f=4$ and 8 are observed when comparing single-star and multiple-star simulations (the shape parameters for single-star simulations ${ }^{25}$ are listed in Table S1 in the Supporting Information). Stars with $f=$ 16 and $f=32$ appear to be more spherical in single-star simulations (resembling octopus-like objects) than when incorporated into a self-assembled object in multiple-star systems. In other words, the intermolecular nanosegregation and the packing of stars in the assembly lead to a more prolate shape in the case of mikto-arm stars with $f=16$ and $f=32$ (compare the values of $a$ and $p$ for these systems in both types of simulations in Tables 1 and $S 1$ of the Supporting Information). The arm length does not seem to have a significant effect on the 

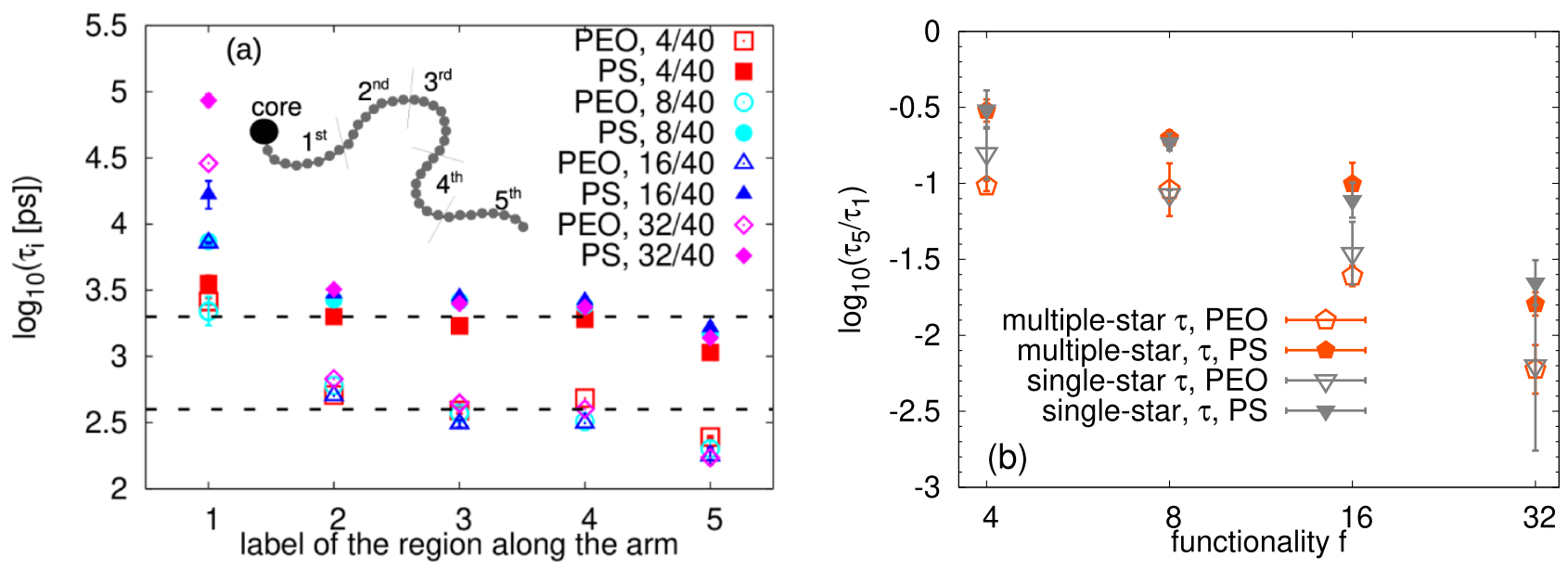

Figure 6. (a) Segmental relaxation times plotted with respect to the position along the arm. The inset shows a schematic illustration of an arm division into five regions. The dashed lines are a guide to the eye and are drawn in line with the plateau in the middle region. (b) Ratio of the segmental times, $\tau_{5} / \tau_{1}$, in the fifth and first region with respect to the star functionality. The error bars were obtained by the standard block averaging method, and for simplicity, only the error bars for the first, third, and fifth region are shown. The rest are of the order of the symbol size.

shape of the individual mikto-arm star in the assembly, and the distribution of asphericities for 16/80 stars reported in ref 24 is very similar to the one presented here for $16 / 40$ stars; however, $16 / 40$ stars are slightly more prolate than $16 / 80$.

A closer inspection of the mikto-arm star configurations in Figure 5a reveals that each star is internally nanosegregated and it consists of segregated PEO and PS regions. In the case of an ideal Janus-like segregation, that is, with each polymer type occupying different "hemispheres" of the molecule, the vectors $\mathbf{R}_{\mathbf{P E O}}$ and $\mathbf{R}_{\mathbf{P S}}$ connecting the center of the star with the segregated domains (see Figure S2a in the Supporting Information and the inset of Figure $5 b$ ) would point to opposite directions, forming an angle $\theta$ of $180^{\circ}$. The normalized distributions of intramolecular angles $\theta$ for our star systems are plotted in Figure $5 b$. For $f \leq 16$, the maximum of the distribution is shifting to larger values of $\theta$ as the number of arms increases, a sign of a more efficient separation of the domains in these nanosegregated molecules. Interestingly, systems which intermolecularly assemble into percolated networks, namely, the $32 / 40$ stars studied in this work and the $16 / 80$ stars from ref 24 , share similar, broader distributions with a maximum positioned at lower values of $\theta$ than the $16 / 40$ stars (see the magenta diamonds and the yellow dashed line in Figure $5 \mathrm{~b}$ ).

The mutual arrangement of $\mathbf{R}_{\mathbf{P E O}}$ and $\mathbf{R}_{\mathbf{P S}}$ vectors in singlestar simulations seems to follow the same trend as the one described for multiple-star simulations above (see Figure S2b in the Supporting Information), except for the mikto-arm star with 32 arms, which has two preferable positions of the domains (i.e., bimodal distribution of $\theta$ ), representing two mutual arrangements of the "head" and "tentacles" in its octopus-like structure. $^{25}$

2.2. Dynamic Properties. To examine the internal local dynamical heterogeneities in the mikto-arm stars, we divide each arm of the star into five regions of eight monomers (see the inset in Figure 6a). In order to study rotational dynamics, we define a backbone vector for PEO and PS components, along each monomer (see Figure S3 in the Supporting Information) and calculate the autocorrelation functions corresponding to those vectors. An example of the fitting procedure of the autocorrelation function together with a detailed description of the procedure for obtaining the relaxation times $\tau_{i}$ for each region can be found in the Supporting Information. We report the average values of the relaxation times, which combine both fitting parameters $\tau_{\mathrm{KWW} i}$ and $\beta_{i}$ in the relation $\tau_{i}=\frac{\tau_{\mathrm{KwW}}}{\beta_{i}} \Gamma\left(\frac{1}{\beta_{i}}\right)$, where $\Gamma$ stands for the gamma function. This relation allows us to account for the broadness of the distribution of relaxation times, for each regime $i$, through the stretching exponent $\beta_{i}$. The average relaxation times for the PEO and the PS segments as a function of the position along the arm (i.e., label $i$ of the region, $i$ $=1,2, \ldots, 5)$ are shown in Figure 6a. The segmental dynamics of the PEO component is faster than that of the PS component (the gap between the two dashed lines in Figure 6a would correspond to the difference in the dynamics of the middle segments) because of very different glass transition temperatures, $T_{\mathrm{g}}$, of PEO and PS, i.e., $T_{\mathrm{g}}(\mathrm{PS})$ is more than $150 \mathrm{~K}$ higher than $T_{\mathrm{g}}(\mathrm{PEO}) .{ }^{43}$ Concerning the dependence of $\tau_{i}$ on the position along the arm, the data for both components show a trend very similar to the one observed in other star-like systems: ${ }^{44,45}$ slow segmental dynamics close to the core (region 1 ), a dynamically homogeneous middle part that is faster than the core region (regions 2, 3, and 4), and an acceleration of the dynamics when approaching the arm end (region 5). The faster dynamics close to the arm end with respect to the middle segments can be explained by a higher configurational freedom and consequent stronger fluctuations of the end monomers. The relaxation time $\tau_{5}$ in this region is almost identical to the average relaxation time found for the oligomeric PEO (o-PEO) chains of the matrix, namely, $\tau(\mathrm{o}-\mathrm{PEO})=224 \mathrm{ps}$ and $\tau_{5}(\mathrm{PEO})$ of the $4 / 40$ star is 246 ps. The relaxation of the segments in region 1 is restrained because of arm attachment to the core. Moreover, their dynamics is also affected by the heterogeneous environment originating from the vicinity of the dissimilar arms connected to the core in the alternating way (Figure 9). This local mixing of the arms close to the core, enforced by the connectivity, led to a coupled dynamics of PEO and PS segments in this region in mikto-arm stars in the polybutadiene matrix. ${ }^{44}$ The actual magnitude of the dynamical retardation in the vicinity of the star core depends both on the star functionality and the monomer type. More specifically, the higher the functionality, the slower is the dynamics in the first region. This phenomenon is attributed to the increase in monomer density close to the core with increasing number of star arms. ${ }^{45,46}$ Concerning the comparison of the two types of arms, the 

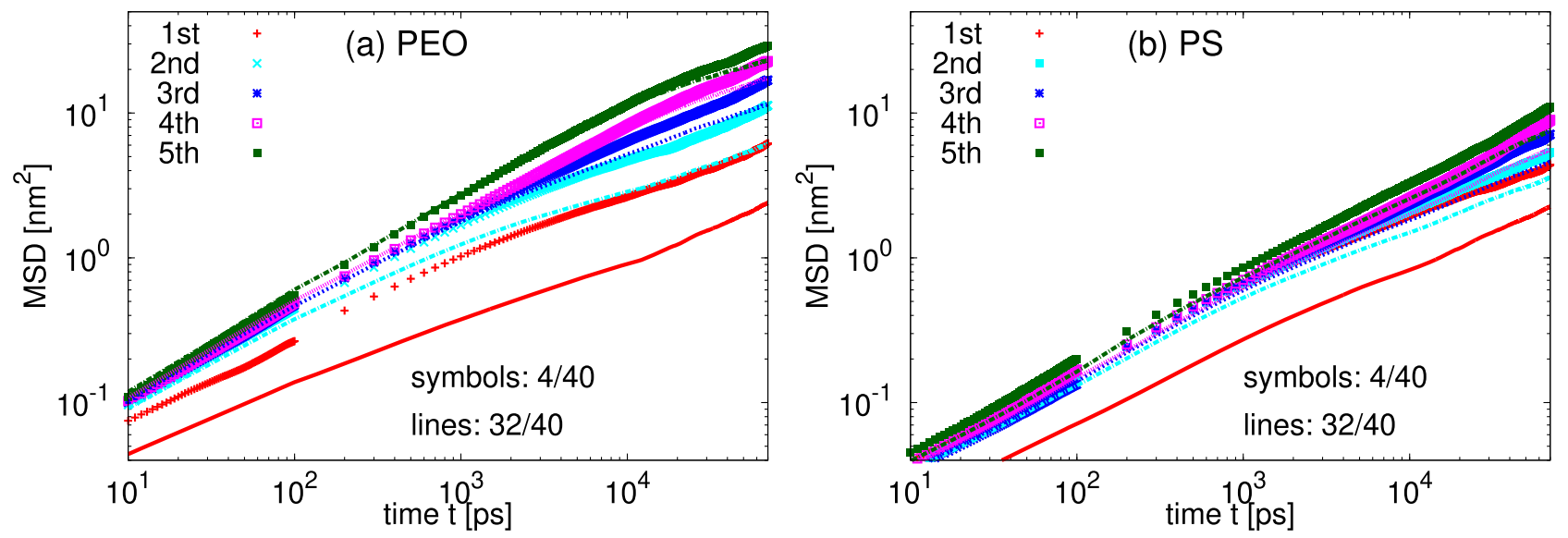

Figure 7. Monomer MSD as a function of time for segments at different regions along the arms for 4/40 (points) and 32/40 stars (lines) at $400 \mathrm{~K}$. (a) Data for PEO arms. (b) Data for PS arms.

internal dynamical heterogeneities are more pronounced in the case of the PEO arms than the PS arms. This is reflected in the plot of the $\tau_{5} / \tau_{1}$ ratio in Figure $6 \mathrm{~b}$, where the values corresponding to the PEO segments are smaller than those for the PS ones, for all studied functionalities. Moreover, in contrast to the results reported from more qualitative, bead-spring models in ref 45 , the data do not seem to follow a specific power law $\tau_{5} / \tau_{1} \sim f^{x}$ in the range of studied functionalities (notice that both axes in Figure $6 \mathrm{~b}$ are in a logarithmic scale).

The segmental dynamics along the arm in mikto-arm stars which are not self-assembled, that is, in single-star simulations, exhibits the same features qualitatively (Figure S4 in the Supporting Information). However, the $\tau_{i}$ of all five PS segments $(i=1, \ldots, 5)$ in the single-star simulations is approximately a factor of 2 faster (compare the filled symbols in Figures 6a and S4 in the Supporting Information). The reason of such differences in the segmental dynamics should be found in the different local environments: in multiple-star systems, PS segments have less (low $T_{\mathrm{g}}$ ) PEO monomers around them, compared to those of single-star ones (see Figure 4b), and thus, they exhibit slower dynamics. Despite the difference in the actual magnitude of the average PS relaxation time, the degree of the intramolecular dynamical heterogeneity (i.e., how much the region close to the star core differs from the arm end) seems to be the same (notice the matching between the gray and orange symbols in Figure 6b).

To study the translational motion, we calculate the monomer mean square displacement (MSD) and average it over the monomers in the given region $i$. The time evolution of the MSDs for the five regions along the arm is shown in Figure 7. In Figure 7 , we compare the data for the mikto-arm stars with functionalities $f=4$ and $f=32$. Focusing first on the PEO arms (Figure 7a), there is an apparent gradient in the mobility of the arm monomers, with the slowest monomers being placed close to the star core. In agreement with the previously described rotational dynamics, the monomers in the first region of 32/40 stars are much slower than those of $4 / 40$ stars, confirming the strong impact of the functionality on the dynamics in this region. In contrast to the common "plateau-like" region for all $f$ observed for $\tau_{i}$ (PEO) of the middle segments $(i=2, \ldots, 4)$, the translational motion of the monomers in the middle part of the arm (second, third, and fourth regions) of 32/40 stars is much slower than those in the middle arm regions of $4 / 40$ stars. The particular time, where the deviation in the dynamics of these monomers is observed, depends on the location of the monomer along the arm, namely, it is $t<10^{2}$ ps for the second region, $t \approx$ $10^{3} \mathrm{ps}$ for the third region, and $t \approx 10^{4} \mathrm{ps}$ for the fourth region (compare symbols and lines for the given region in Figure $7 \mathrm{a}$ ). The MSD data for the end monomers, fifth region, overlap, and only small deviations between $4 / 40$ and $32 / 40$ stars are observed in the end of the simulated time window, where the data are very noisy.

The situation is different for the PS monomers (see Figure $7 \mathrm{~b})$ : the monomers in all regions along the arm are slower in the $32 / 40$ mikto-arm stars in comparison to the 4/40 stars (compare lines with symbols in Figure $7 \mathrm{~b}$ ). We would like to point out that such mobility gradients along the arm have been also observed in atomistic model systems of self-assembled $16 / 80$ mikto-arm stars, ${ }^{24}$ as well as in bead-spring simulations of homopolymer star melts with low functionality $(f<6),{ }^{47,48}$ and they were attributed to the star-like architecture.

In Figure S5 of the Supporting Information, we compare the MSD data for 32/40 mikto-arm stars studied here (multiple-star simulations, points) with the data obtained for simulations of a single $32 / 40$ star in the o-PEO matrix (single-star simulations, lines). The curves for PEO monomers (Figure S5a) in all arm regions in both types of simulations overlap, and the monomers in the self-assembled stars move slower only after the time scales $t>4 \mathrm{~ns}$. The time evolutions of MSDs belonging to the PS arms (Figure S5b) diverge in the whole simulated time window, and the dynamics of the PS component in the single-star surrounded by o-PEO chains is faster than the dynamics of PS monomers assembled into the percolated network. This observation highlights the importance of the local environment in the dynamical response of the material, that is, the fact that the PS monomers of self-assembled stars are able to avoid the PEO/PS interactions by forming intermolecular assemblies, while the only way to avoid unfavorable interactions in a single-star system is internal nanosegregation of the PS arms within the star (see Figure 4 and the related discussion). In the latter case, the PS arms are more "exposed", which make them more "vulnerable" to the dynamical coupling with the faster o-PEO matrix. This explains also the lower values of $\tau_{i}(\mathrm{PS})$ in the single-star simulations in all regions $(i=1, \ldots, 5)$ discussed above.

To further quantify the magnitude of the internal heterogeneities, related to the monomer translational mobility, we compute a ratio of characteristic relaxation times, a quantity analogous to the ratio $\tau_{5} / \tau_{1}$ presented in the analysis of the 

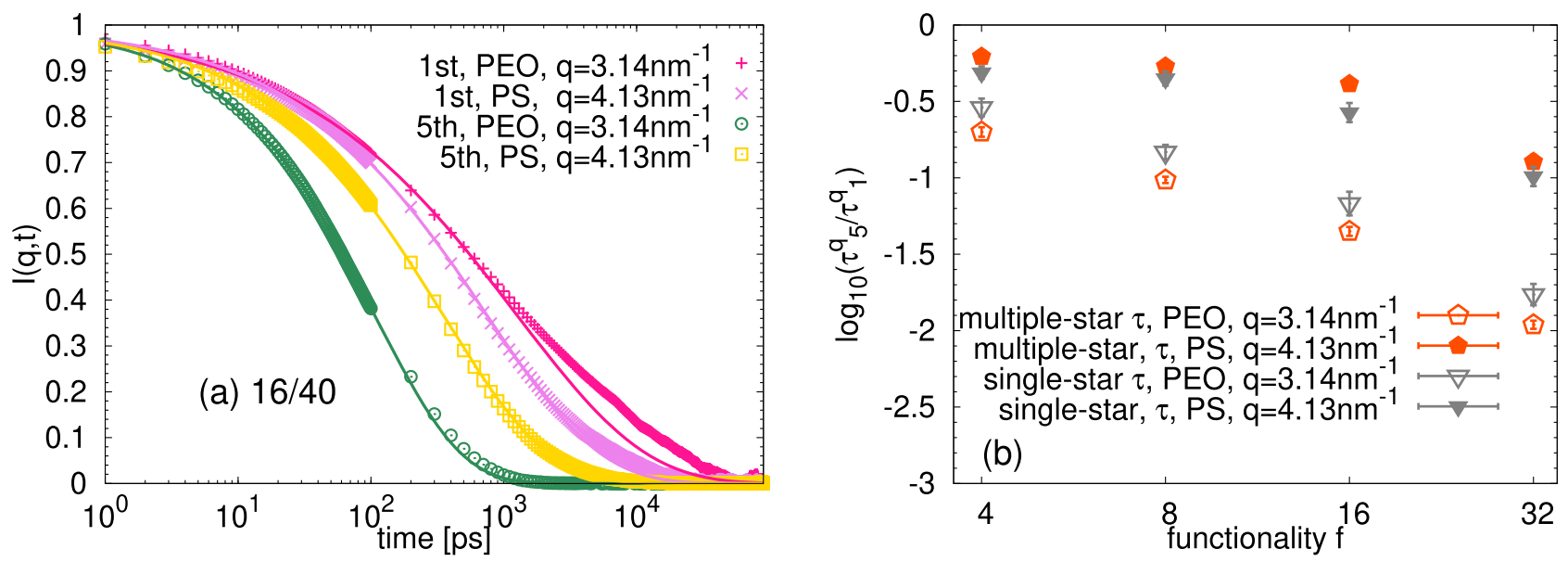

Figure 8. (a) Incoherent dynamical structure factor $I(q, t)$ for the atoms in the first and in fifth region placed on the arms of 16/40 mikto-arm stars for wavevectors that correspond to the end-to-end distance of an 8-mer of PEO or PS units, respectively. The solid lines represent the fits to the stretched exponential functions. (b) Ratio of the average characteristic times, $\tau_{5}{ }^{q} / \tau_{1}^{q}$, obtained from the fitting procedure in (a) with respect to the star functionality. The error bars were obtained by the standard block averaging method.

rotational dynamics. To achieve this, we first calculated the incoherent dynamical structure factor for each region, $i$ (i.e., from 1 to 5) on the PEO and PS arms, via the following equation

$$
\begin{aligned}
I(\mathbf{q}, t) & =\frac{1}{M_{i}}\left\langle\sum_{k}^{M_{i}} \exp \left(i \mathbf{q} \cdot\left(\mathbf{r}_{k}\left(t+t_{0}\right)-\mathbf{r}_{k}\left(t_{0}\right)\right)\right\rangle\right. \\
& =\left\langle\sum_{k}^{M_{i}} \frac{\sin q\left|\mathbf{r}_{k}\left(t+t_{0}\right)-\mathbf{r}_{k}\left(t_{0}\right)\right|}{q\left|\mathbf{r}_{k}\left(t+t_{0}\right)-\mathbf{r}_{k}\left(t_{0}\right)\right|}\right\rangle
\end{aligned}
$$

where $M_{i}$ denotes the number of united atoms in the given region $i, \mathbf{r}_{k}$ is the position vector of the atom at the given time, $\boldsymbol{q}$ is the wavevector and $q$ its magnitude. $I(q, t)$ provides information on different length scales, depending on the value of $q$. In order to study the translational dynamics on length scales relevant to the size of the selected arm region, we calculated $I(\mathbf{q}$, $t$ ) for two values of $q$, which were obtained as: $q=2 \pi / r_{\mathrm{e} i}$, where $r_{\mathrm{e} i}$ is the average distance from the first to the last atom in the given region. As each studied region on an arm is eight monomers long, $r_{\mathrm{e} i}$ would approximately correspond to the "end-to-end" distance of the eight monomeric units of the specific polymer type, that is, either PEO or PS. Then, we fitted the obtained $I(\mathbf{q}, t)$ for each region and for the given $q$ (i.e., 3.14 $\mathrm{nm}^{-1}$ for PEO and $4.13 \mathrm{~nm}^{-1}$ for the PS arms) with the stretched exponential function, in the same way as described above for the rotational dynamics. One example of the $I(\mathbf{q}, t)$ data for the first and fifth region in 16/40 stars is presented in Figure 8a together with the corresponding fits. The ratio $\tau_{5}{ }^{q} / \tau_{1}^{q}$ of the so-obtained characteristic times for the fifth and first region is plotted as a function of $f$ in Figure $8 \mathrm{~b}$. Similarly to $\tau_{5} / \tau_{1}$ obtained from the backbone vector correlation function, this quantity decays with increasing functionality and the differences in dynamics along the arm are more pronounced for the PEO than for the PS arms. The data from the single-star simulations copy the tendency of those from the multiple-star simulations closely, confirming that the degree of internal heterogeneity along the arm is mostly determined by the star-like architecture and less by the local environment in the self-assembling process. Comparing the actual values, the differences in dynamics of the two extremes of the star arm seem to be more pronounced in rotational than in translational motion (compare the values in Figure $8 \mathrm{~b}$ with those in Figure 6b). However, we would like to stress that for such a direct comparison of these two measured quantities, one should consider their different natures. More specifically, the decay of the autocorrelation function of the backbone vector (defined and shown in the Supporting Information) is dominated by the slow elements, whereas the displacement $\left(\mathbf{r}_{k}\left(t+t_{0}\right)-\mathbf{r}_{k}\left(t_{0}\right)\right)$ in eq 1 is dictated by the motion of the fast particles, especially at early times. Moreover, the actual values of $\tau_{1}{ }^{q}$ and $\tau_{5}{ }^{q}$ depend on the chosen $q$, in other words, on the chosen length scale under study.

\section{CONCLUSIONS}

In this work, we have used atomistic molecular dynamics simulations to study symmetric amphiphilic mikto-arm stars, consisting of varying number of PS and PEO arms, in a selective polymer matrix. The internal nanosegregation of the immiscible star arms together with the selective character of the polymer matrix determines the structural and dynamical behavior of the studied systems.

The stars with the functionality $f \leq 16$ resemble prolate Januslike particles and self-assemble into cylindrical objects. The mikto-arm stars with $f=32$ are the most spherical and the ones mostly affected by the geometric constrains related to the higher number of arms. More specifically, because of the high functionality, these star-like systems are not able to efficiently reduce the unfavorable PEO/PS contacts, and consequently, they self-assemble into a percolated network. Moreover, the local density profile analysis shows a more homogeneous environment for the PS arms segregated into a multiple-star selfassembled structure (i.e., the unfavorable PEO/PS contacts are reduced) compared to the single-star systems.

The internal dynamical heterogeneities in the translational motion of the monomers induced by the star-like architecture are amplified with increasing star functionality and are more pronounced in the PEO than in the PS arms. A similar trend is observed for rotational dynamics; however, the dynamics is affected only in the region in the vicinity of the core. The differences in dynamics of the two extremes of the star arm (i.e., the one in the center of the star and the other on the arm end) seem to be mostly governed by the star-like architecture and less affected by the changing local environment in the selfassembling process. 


\section{COMPUTATIONAL METHODS}

Because of the diverse structural and dynamical features of the blend components, particular attention was paid to the preparation and equilibration of the systems. The full description of the used protocol can be found elsewhere; ${ }^{24}$ here, we present its shorter version. The simulations were performed with GROMACS package ${ }^{49}$ using the united-atom model of the TraPPE force field. ${ }^{50-52}$ The united-atom approach means that the hydrogens are not simulated explicitly, but they are accounted for together with the carbon atom in one united-atom unit, that is, $\mathrm{CH}_{2}$. According to the TraPPE force field, the PEO-united atoms carry partial charges and their 1-4 Coulomb interactions are rescaled with a prefactor of $0.5 .^{52}$ The PS-united atoms are neutral, that is, they do not contribute to the total electrostatic energy. We simulated atactic PS, and, in order to keep the aromatic rings planar, the harmonic type of improper dihedral potential was used. ${ }^{49,51}$ In the PS arms, the nonbonded 1-4 interactions were set to 0 .

Each star consists of a central core and $f$ arms attached to it: $f$ / 2 arms consist of united atoms belonging to the PEO polymer type and $f / 2$ arms consist of united atoms belonging to the PS polymer type. The star cores have a dendritic architecture and are built by carbon units (i.e., by united atoms representing $\mathrm{C}$, $\mathrm{CH}$, or $\mathrm{CH}_{2}$ groups of atoms). The number of the core units varies from $5(f=4)$ to $61(f=32)$. The PEO and PS arms are attached alternately to the core (see Figure 9), and the initial

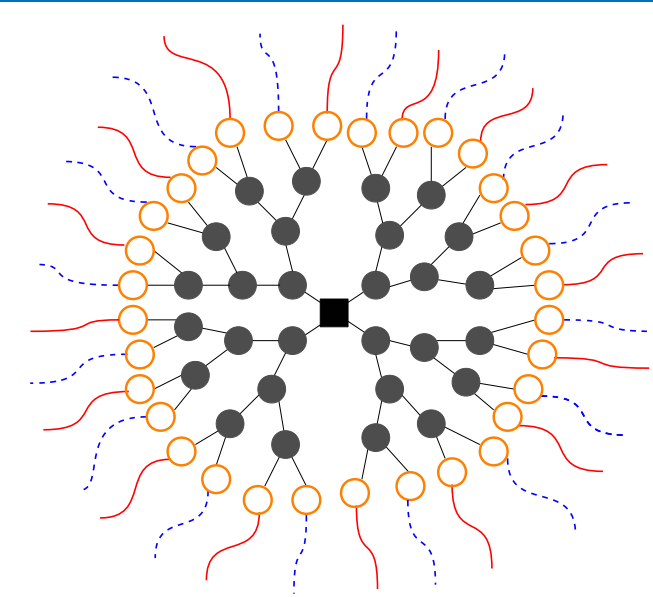

Figure 9. Scheme of the alternating attachment of the arms to the core of a 32/40 star; the blue dashed lines represent the connected PS arms, and the red solid lines represent the PEO arms. The filled gray circles represent $\mathrm{CH}$ carbon units, the empty orange ones represent $\mathrm{CH}_{2}$ units, and the black square is the middle carbon.

configuration was prepared with the arms fully stretched. An energy minimization and series of short runs with reduced time step ( $0.1 \mathrm{fs})$ with bonds constrained by the LINCS algorithm ${ }^{53}$ were performed to reduce the number of possible overlaps in the initial star configuration without causing stretching of the bonds. Once the single-star configuration was free of overlaps, 10 stars and the corresponding number of linear o-PEO chains were placed randomly into a box with a size much bigger than the actual size at the desired density. Then, we run an NPT simulation under a pressure of $1 \mathrm{~atm}$ maintained by a Berendsen barostat and with the cutoff method for the Coulomb interactions. Once we achieved the desired density, we continued the equilibration for another 70-100 ns. After the first equilibration, we constrained the bonds by the LINCS algorithm and performed a heating procedure (Langevin dynamics, $700 \mathrm{~K}, 50 \mathrm{~ns}$ ). This part of the equilibration resembles experimental protocols, where the material is often heated and cooled down to assure a proper preparation of the sample. Next and prior to the production run, we switched to the Nose-Hoover thermostat in combination with the ParrinelloRahman barostat and simulated the system under the given temperature $(400 \mathrm{~K})$ and pressure of $1 \mathrm{~atm}$ for $100 \mathrm{~ns}$.

The temperature of the production runs, $400 \mathrm{~K}$, was kept constant by applying the Nose-Hoover thermostat, a pressure of $1 \mathrm{~atm}$ was maintained using the Parrinello-Rahman barostat, and the long-ranged electrostatic interactions between the partially charged PEO units were calculated by the particle-mesh Ewald method. Each component of the system (PEO arms, PS arms, and o-PEO) was coupled to a separate thermostat to prevent a creation of stationary temperature gradients. ${ }^{24,54}$ The time step used in the production runs is $1 \mathrm{fs}$, and their length is $200 \mathrm{~ns}$ for the 4/40, $135 \mathrm{~ns}$ for the 8/40, $145 \mathrm{~ns}$ for the $16 / 40$, and 124 ns for the $32 / 40$ system.

\section{ASSOCIATED CONTENT}

\section{Supporting Information}

The Supporting Information is available free of charge at https://pubs.acs.org/doi/10.1021/acsomega.0c04167.

Equations for the radius of gyration tensor and shape parameters, table with the shape properties of stars in single-star simulations, definitions of intermolecular and intramolecular angles, procedure for obtaining segmental relaxation times, and additional results from the singlestar simulations (PDF)

\section{AUTHOR INFORMATION}

\section{Corresponding Authors}

Petra Bačová - Institute of Applied and Computational Mathematics (IACM), Foundation for Research and Technology Hellas (FORTH), GR-70013 Heraklion, Crete, Greece; Email: pbacova@iacm.forth.gr

Vagelis Harmandaris - Institute of Applied and Computational Mathematics (IACM), Foundation for Research and Technology Hellas (FORTH), GR-70013 Heraklion, Crete, Greece; Department of Mathematics and Applied Mathematics, University of Crete, GR-70013 Heraklion, Crete, Greece; Computation-Based Science and Technology Research Center, The Cyprus Institute, 2121 Nicosia, Cyprus; ○ orcid.org/0000-0002-9613-7639; Email: harman@ uoc.gr

\section{Authors}

Emmanouil Glynos - Institute of Electronic Structure and Laser, Foundation for Research and Technology Hellas (FORTH), GR-70013 Heraklion, Crete, Greece; () orcid.org/0000-0002-0623-8402

Spiros H. Anastasiadis - Institute of Electronic Structure and Laser, Foundation for Research and Technology Hellas (FORTH), GR-70013 Heraklion, Crete, Greece; Department of Chemistry, University of Crete, GR-70013 Heraklion, Crete, Greece; 이이이.org/0000-0003-0936-1614

Complete contact information is available at: https://pubs.acs.org/10.1021/acsomega.0c04167

\section{Notes}

The authors declare no competing financial interest. 


\section{ACKNOWLEDGMENTS}

P.B. and E.G. acknowledge the financial support of the Stavros Niarchos Foundation within the framework of the project ARCHERS ("Advancing Young Researchers' Human Capital in Cutting Edge Technologies in the Preservation of Cultural Heritage and the Tackling of Societal Challenges"). V.H. acknowledges support by the project "SimEA", funded by the European Union's Horizon 2020 research and innovation program under grant agreement no. 810660 .

\section{REFERENCES}

(1) Aricò, A. S.; Bruce, P.; Scrosati, B.; Tarascon, J.-M.; van Schalkwijk, W. Nanostructured Materials for Advanced Energy Conversion and Storage Devices. Nat. Mater. 2005, 4, 366-377.

(2) Behl, M.; Razzaq, M. Y.; Lendlein, A. Multifunctional ShapeMemory Polymers. Adv. Mater. 2010, 22, 3388-3410.

(3) Glotzer, S. C.; Solomon, M. J. Anisotropy of Building Blocks and Their Assembly Into Complex Structures. Nat. Mater. 2007, 6, 557562.

(4) Li, Z.-W.; Lu, Z.-Y.; Sun, Z.-Y.; An, L.-J. Model, self-assembly structures, and phase diagram of soft Janus particles. Soft Matter 2012, 8, 6693-6697.

(5) Percebom, A. M.; Costa, L. H. M. Formation and assembly of amphiphilic Janus nanoparticles promoted by polymer interactions. Adv. Colloid Interface Sci. 2019, 269, 256-269.

(6) Pons-Siepermann, I. C.; Glotzer, S. C. Design of patchy particles using ternary self-assembled monolayers. Soft Matter 2012, 8, 62266231.

(7) Rovigatti, L.; Capone, B.; Likos, C. N. Soft self-assembled nanoparticles with temperature-dependent properties. Nanoscale 2016, $8,3288-3295$.

(8) Bianchi, E.; van Oostrum, P. D. J.; Likos, C. N.; Kahl, G. Inverse patchy colloids: Synthesis, modeling and self-organization. Curr. Opin. Colloid Interface Sci. 2017, 30, 8-15.

(9) Deng, R.; Liang, F.; Zhu, J.; Yang, Z. Recent advances in the synthesis of Janus nanomaterials of block copolymers. Mater. Chem. Front. 2017, 1, 431-443.

(10) Gröschel, A. H.; Müller, A. H. E. Self-assembly concepts for multicompartment nanostructures. Nanoscale 2015, 7, 11841-11876.

(11) Gröschel, A. H.; Walther, A.; Löbling, T. I.; Schmelz, J.; Hanisch, A.; Schmalz, H.; Müller, A. H. E. Facile, Solution-Based Synthesis of Soft, Nanoscale Janus Particles with Tunable Janus Balance. J. Am. Chem. Soc. 2012, 134, 13850-13860.

(12) Bozorgui, B.; Meng, D.; Kumar, S. K.; Chakravarty, C.; Cacciuto, A. Fluctuation-Driven Anisotropic Assembly in Nanoscale Systems. Nano Lett. 2013, 13, 2732-2737.

(13) Akcora, P.; Liu, H.; Kumar, S. K.; Moll, J.; Li, Y.; Benicewicz, B. C.; Schadler, L. S.; Acehan, D.; Panagiotopoulos, A. Z.; Pryamitsyn, V.; Ganesan, V.; Ilavsky, J.; Thiyagarajan, P.; Colby, R. H.; Douglas, J. F. Anisotropic self-assembly of spherical polymer-grafted nanoparticles. Nat. Mater. 2009, 8, 354-359.

(14) Zhao, B.; Zhu, L. Mixed Polymer Brush-Grafted Particles: A New Class of Environmentally Responsive Nanostructured Materials. Macromolecules 2009, 42, 9369-9383.

(15) Chen, C.; Tang, P.; Qiu, F. Binary Hairy Nanoparticles: Recent Progress in Theory and Simulations. J. Polym. Sci., Part B: Polym. Phys. 2014, 52, 1583-1599.

(16) Borisov, O. V.; Zhulina, E. B. Theory of self-assembly of triblock ter-polymers in selective solvent towards corona-compartmentalized (Janus) micelles. Polymer 2013, 54, 2043-2048.

(17) Zhulina, E. B.; Borisov, O. V. Effect of Block Copolymer Architecture on Morphology of Self-Assembled Aggregates in Solution. ACS Macro Lett. 2013, 2, 292-295.

(18) Chang, Y.; Chen, W.-C.; Sheng, Y.-J.; Jiang, S.; Tsao, H.-K. Intramolecular Janus Segregation of a Heteroarm Star Copolymer. Macromolecules 2005, 38, 6201-6209.
(19) Hebbeker, P.; Plamper, F. A.; Schneider, S. Effect of the Molecular Architecture on the Internal Complexation Behavior of Linear Copolymers and Miktoarm Star Polymers. Macromol. Theory Simul. 2015, 24, 110-116.

(20) Hebbeker, P.; Steinschulte, A. A.; Schneider, S.; Plamper, F. A. Balancing Segregation and Complexation in Amphiphilic Copolymers by Architecture and Confinement. Langmuir 2017, 33, 4091-4106.

(21) Hebbeker, P.; Steinschulte, A. A.; Schneider, S.; Okuda, J.; Möller, M.; Plamper, F. A.; Schneider, S. Complexation in Weakly Attractive Copolymers with Varying Composition and Topology: Linking Fluorescence Experiments and Molecular Monte Carlo Simulations. Macromolecules 2016, 49, 8748-8757.

(22) Gârlea, I. C.; Bianchi, E.; Capone, B.; Rovigatti, L.; Likos, C. N. Hierarchical self-organization of soft patchy nanoparticles into morphologically diverse aggregates. Curr. Opin. Colloid Interface Sci. 2017, 30, 1-7.

(23) Moghimi, E.; Chubak, I.; Statt, A.; Howard, M. P.; Founta, D.; Polymeropoulos, G.; Ntetsikas, K.; Hadjichristidis, N.; Panagiotopoulos, A. Z.; Likos, C. N.; Vlassopoulos, D. SelfOrganization and Flow of Low-Functionality Telechelic Star Polymers with Varying Attraction. ACS Macro Lett. 2019, 8, 766-772.

(24) Bačová, P.; Foskinis, R.; Glynos, E.; Rissanou, A. N.; Anastasiadis, S. H.; Harmandaris, V. Effect of macromolecular architecture on the self-assembly behavior of copolymers in a selective polymer host. Soft Matter 2018, 14, 9562-9570.

(25) Bačová, P.; Glynos, E.; Anastasiadis, S. H.; Harmandaris, V. Nanostructuring Single-Molecule Polymeric Nanoparticles via Macromolecular Architecture. ACS Nano 2019, 13, 2439-2449.

(26) Glynos, E.; Papoutsakis, L.; Pan, W.; Giannelis, E. P.; Nega, A. D.; Mygiakis, E.; Sakellariou, G.; Anastasiadis, S. H. Nanostructured Polymer Particles as Additives for High Conductivity, High Modulus Solid Polymer Electrolytes. Macromolecules 2017, 50, 4699-4706.

(27) Pavlopoulou, E.; Anastasiadis, S. H.; Iatrou, H.; Moshakou, M.; Hadjichristidis, N.; Portale, G.; Bras, W. Micellization of Miktoarm Star $\mathrm{S}_{\mathrm{n}} \mathrm{I}_{\mathrm{n}}$ Copolymers in Block Copolymer/Homopolymer Blends. Macromolecules 2009, 42, 5285-5295.

(28) Gohy, J.-F. Block Copolymer Micelles. In Block Copolymers II; Abetz, V., Ed.; Springer Berlin Heidelberg: Berlin, Heidelberg, 2005; pp $65-136$.

(29) Retsos, H.; Anastasiadis, S. H.; Pispas, S.; Mays, J. W.; Hadjichristidis, N. Interfacial Tension in Binary Polymer Blends in the Presence of Block Copolymers. 2. Effects of Additive Architecture and Composition. Macromolecules 2004, 37, 524-537.

(30) Pispas, S.; Hadjichristidis, N.; Potemkin, I.; Khokhlov, A. Effect of Architecture on the Micellization Properties of Block Copolymers: A2B Miktoarm Stars vs AB Diblocks. Macromolecules 2000, 33, 17411746.

(31) Sotiriou, K.; Nannou, A.; Velis, G.; Pispas, S. Micellization Behavior of PS(PI)3 Miktoarm Star Copolymers. Macromolecules 2002, 35, 4106-4112.

(32) Pispas, S.; Poulos, Y.; Hadjichristidis, N. Micellization Behavior of (PS)8(PI)8 Miktoarm (Vergina) Star Copolymers. Macromolecules 1998, 31, 4177-4181.

(33) Pispas, S.; Avgeropoulos, A.; Hadjichristidis, N.; Roovers, J. Hydrodynamic properties of $\mathrm{A}_{8} \mathrm{~B}_{8}$ type miktoarm (Vergina) stars. J. Polym. Sci., Part B: Polym. Phys. 1999, 37, 1329-1335.

(34) Havránková, J.; Limpouchová, Z.; Stěpánek, M.; Procházka, K. Self-Assembly of Heteroarm Star Copolymers - A Monte Carlo Study. Macromol. Theory Simul. 2007, 16, 386-398.

(35) Lin, C.-M.; Chen, Y.-Z.; Sheng, Y.-J.; Tsao, H.-K. Effects of macromolecular architecture on the micellization behavior of complex block copolymers. React. Funct. Polym. 2009, 69, 539-545. , Special Issue: Block Copolymers

(36) Nardai, M. M.; Zifferer, G. Concentration Dependence of Size, Shape, and Orientation of Copolymers, 1 - Linear Diblock and 4-arm Hetero Star Polymers. Macromol. Theory Simul. 2011, 20, 675-689.

(37) Li, B.; Zhao, L.; Qian, H. J.; Lu, Z. Y. Coarse-grained simulation study on the self-assembly of miktoarm star-like block copolymers in various solvent conditions. Soft Matter 2014, 10, 2245-2252. 
(38) Li, B.; Zhu, Y.-L.; Liu, H.; Lu, Z.-Y. Brownian dynamics simulation study on the self-assembly of incompatible star-like block copolymers in dilute solution. Phys. Chem. Chem. Phys. 2012, 14, 49644970.

(39) Havránková, J.; Limpouchová, Z.; Procházka, K. Monte Carlo Study of Heteroarm Star Copolymers in Good and Selective Solvents. Macromol. Theory Simul. 2003, 12, 512-523.

(40) Vlahos, C. H.; Horta, A.; Hadjichristidis, N.; Freire, J. J. Monte Carlo Calculations of AxBf-x Miktoarm Star Copolymers. Macromolecules 1995, 28, 1500-1505.

(41) Rubio, A. M.; Brea, P.; Freire, J. J.; Vlahos, C. Sizes and Second Virial Coefficients of Miktoarm Star Polymers. Macromolecules 2000, 33, 207-216.

(42) Kalyuzhnyi, O.; Ilnytskyi, J. M.; Holovatch, Y.; von Ferber, C. Universal shape characteristics for the mesoscopic star-shaped polymer via dissipative particle dynamics simulations. J. Phys.: Condens. Matter 2018, 30, 215101.

(43) Bicerano, J. Glass Transition. Encyclopedia of Polymer Science and Technology; American Cancer Society, 2001.

(44) Bačová, P.; Glynos, E.; Anastasiadis, S. H.; Harmandaris, V. Spatio-temporal heterogeneities in nanosegregated single-molecule polymeric nanoparticles. Soft Matter 2020, 16, 4584-4590.

(45) Chremos, A.; Glynos, E.; Green, P. F. Structure and dynamical intra-molecular heterogeneity of star polymer melts above glass transition temperature. J. Chem. Phys. 2015, 142, 044901.

(46) Grest, G. S.; Fetters, L. J.; Huang, J. S.; Richter, D. Star Polymers: Experiment, Theory, and Simulation. Advances in Chemical Physics; John Wiley \& Sons, Inc., 2007; pp 67-163.

(47) Bačová, P.; Hawke, L. G. D.; Read, D. J.; Moreno, A. J. Dynamics of Branched Polymers: A Combined Study by Molecular Dynamics Simulations and Tube Theory. Macromolecules 2013, 46, 4633-4650.

(48) Holler, S.; Moreno, A. J.; Zamponi, M.; Bačová, P.; Willner, L.; Iatrou, H.; Falus, P.; Richter, D. The Role of the Functionality in the Branch Point Motion in Symmetric Star Polymers: A Combined Study by Simulations and Neutron Spin Echo Spectroscopy. Macromolecules 2018, 51, 242-253.

(49) Hess, B.; Kutzner, C.; van der Spoel, D.; Lindahl, E. GROMACS 4: Algorithms for Highly Efficient, Load-Balanced, and Scalable Molecular Simulation. J. Chem. Theory Comput. 2008, 4, 435-447.

(50) Martin, M. G.; Siepmann, J. I. Transferable Potentials for Phase Equilibria. 1. United-Atom Description of n-Alkanes. J. Phys. Chem. B 1998, 102, 2569-2577.

(51) Wick, C. D.; Martin, M. G.; Siepmann, J. I. Transferable Potentials for Phase Equilibria. 4. United-Atom Description of Linear and Branched Alkenes and Alkylbenzenes. J. Phys. Chem. B 2000, 104, $8008-8016$

(52) Fischer, J.; Paschek, D.; Geiger, A.; Sadowski, G. Modeling of Aqueous Poly(oxyethylene) Solutions: 1. Atomistic Simulations. J. Phys. Chem. B 2008, 112, 2388-2398.

(53) Hess, B.; Bekker, H.; Berendsen, H. J. C.; Fraaije, J. G. E. M. LINCS: A linear constraint solver for molecular simulations. J. Comput. Chem. 1997, 18, 1463-1472.

(54) Lingenheil, M.; Denschlag, R.; Reichold, R.; Tavan, P. The "HotSolvent/Cold-Solute" Problem Revisited. J. Chem. Theory Comput. 2008, 4, 1293-1306. 